\title{
Demonstration of the Lateral Casimir Force
}

\author{
F. Chen, U. Mohideen*, \\ Department of Physics, University of California, Riverside, California 92521 \\ G. L. Klimchitskaya ${ }^{\dagger}$ and V. M. Mostepanenko ${ }^{\ddagger}$ \\ Departamento de Física, Universidade Federal da Paraíba, C.P. 5008, CEP 58059-970, João Pessoa, Pb-Brazil
}

The lateral Casimir force between a sinusoidally corrugated gold coated plate and large sphere was measured for surface separations between $0.2 \mu \mathrm{m}$ to $0.3 \mu \mathrm{m}$ using an atomic force microscope. The measured force shows the required periodicity corresponding to the corrugations. It also exhibits the necessary inverse fourth power distance dependence. The obtained results are shown to be in good agreement with a complete theory taking into account the imperfectness of the boundary metal. This demonstration opens new opportunities for the use of the Casimir effect for lateral translation in microelectromechanical systems.

PACS numbers: 12.20.Fv, 42.50.Lc, 61.16.Ch

The archetypical Casimir force [1] leads to an attraction between two neutral metal plates placed in vacuum. The force results from the alteration by the metal boundaries of the zero point electromagnetic energy which is present in empty space. Uniquely the Casimir force is independent of the electric charge and other interaction constants. As a result, it is strongly dependent on geometry and topology of the boundary and can be both attractive as well as repulsive (for a review of different aspects of the Casimir effect see monographs [2, 3]).

The normal Casimir force which leads to an attraction perpendicular to the two surfaces has been demonstrated first between flat plates [4] and between a flat plate and a lens [5, [. the normal Casimir force between a large sphere and a flat plate has been performed with increased precision $[7$ 9. Also the non-trivial boundary dependence of the Casimir force acting between a large sphere and plate with periodic uniaxial sinusoidal corrugations was demonstrated in Ref. [10]. This has led to extensive theoretical study of the corrections to the Casimir effect due to various factors as finite conductivity of the boundary metal, surface roughness, and nonzero temperature. Also the combined effect of these factors was investigated (new experimental and theoretical developments are presented in review [11]). Even more importantly, the Casimir effect is finding new applications in fundamental science and engineering. Thus, with the advent of modern unified theories involving compact dimensions, precision measurements of the Casimir force have been used to set limits on the presence of hypothetical forces 12 14]. With regard to technological applications, both static and dynamic microelectromechanical machines have been created actuated by the normal Casimir force 15] 16]. Also, adhesion and sticking of moving parts in microelectromechanical systems due to the Casimir effect were investigated [17].

In this letter we report the first demonstration of the lateral Casimir force. It acts between two aligned corrugated surfaces and leads to a mechanical force acting tangential to the surfaces. Similar to the normal Casimir force, the lateral Casimir force also originates from the modifications of electromagnetic zero point oscillations. The possibility of a lateral Casimir force for anisotropic boundaries was investigated theoretically and the harmonic dependence on a correspondent angle was predicted (see [3, 18, 19] for the plates made of anisotropic material and [20 22] for the corrugated plates made of ideal metal). Note that other motional frictional forces between two flat parallel surfaces have been suggested but they would be several orders of magnitude smaller [23,24]. Here we compare the experimental data with a complete theory taking into account the finite conductivity corrections of the boundary metal. The predicted sinusoidal dependence of the lateral force on the phase shift and inverse fourth power dependence on surface separation is confirmed and good agreement with a complete theory is demonstrated. This demonstration opens new opportunities for the actuation of lateral motion in microelectromechanical systems based entirely on the vacuum effects of quantum electrodynamics.

The regularized zero point energy per unit area for two parallel plates of infinite conductivity a distance $z$ apart is given by $U(z)=-\pi^{2} \hbar c /\left(720 z^{3}\right)$ 值 3.11. This results in the normal Casimir force per unit area $F=-\partial U / \partial z=$ $-\pi^{2} \hbar c /\left(240 z^{4}\right)$. As metals have only a finite conductivity, corresponding corrections can be incorporated in terms of the plasma wavelength $\lambda_{p}$ leading to 25 27]

\footnotetext{
${ }^{*}$ Corresponding author. E-mail: umar.mohideen@ucr.edu

${ }^{\dagger}$ On leave from North-West Polytechnical University,

St.Petersburg, Russia.

${ }^{\ddagger}$ On leave from Research and Innovation Enterprise "Modus", Moscow, Russia.
} 


$$
E_{p p}(z)=-\frac{\pi^{2} \hbar c}{720 z^{3}}\left[1+\sum_{n=1}^{4} c_{n}\left(\frac{\lambda_{p}}{2 \pi z}\right)^{n}\right]
$$

where the coefficients are $c_{1}=-4, c_{2}=72 / 5$,

$$
c_{3}=-\frac{320}{7}\left(1-\frac{\pi^{2}}{210}\right), \quad c_{4}=\frac{400}{3}\left(1-\frac{163 \pi^{2}}{7350}\right) .
$$

Eq.(11) is applicable at the separations $z \geq \lambda_{p}$ with an accuracy of about (1-2)\%.

Now let us consider plates covered by the longitudinal corrugations described by the functions

$$
z_{1}=A_{1} \sin (2 \pi x / \Lambda), \quad z_{2}=z+A_{2} \sin (2 \pi x / \Lambda+\varphi)
$$

where $z$ is the mean separation distance between the two surfaces, $\Lambda$ is the corrugation period, $A_{1}$ and $A_{2}$ are the corrugation amplitudes.

The normal separation distance between two points of the corrugated surfaces can be represented as

$$
z_{2}-z_{1}=z+b \cos (2 \pi x / \Lambda-\alpha)
$$

where

$$
\begin{aligned}
& b=b(\varphi)=\left(A_{1}^{2}+A_{2}^{2}-2 A_{1} A_{2} \cos \varphi\right)^{1 / 2} \\
& \tan \alpha=\left(A_{2} \cos \varphi-A_{1}\right) /\left(A_{2} \sin \varphi\right) .
\end{aligned}
$$

For corrugated plates with large corrugation period $(\Lambda>z)$ the Casimir energy density can be found by the additive summation of the results obtained for plane plates (see Eq. (11)) using the fact that all the separation distances $z_{2}-z_{1}$ given by Eq. (3) are equally probable [11,22,28]

$$
E_{p p}^{c o r}(z, \varphi)=\frac{1}{\Lambda} \int_{0}^{\Lambda} E_{p p}\left(z_{2}-z_{1}\right) d x .
$$

Experimentally it is hard to maintain two parallel plates uniformly separated by distances less than a micron. So one of the plates is replaced by a metal sphere of radius $R$ where $R \gg z$. Here a sphere of radius $R=100 \mu$ m imprinted by sinusoidal corrugations was used instead of one of the plates (see Fig. 1). As both $z \ll R$ and $\Lambda \ll R$, the normal Casimir force can be calculated by use of the Proximity Force Theorem [29] as $F^{n o r}(z, \varphi)=2 \pi R E_{p p}^{c o r}(z, \varphi)$. The accuracy of this theorem for our parameters is of order $0.2 \%$ (note that the recent result [30] claiming a worse accuracy for the PFT is applicable only to the pure nonrelativistic regime with separations $z$ no larger than several nanometers and also small $R$ ). By integrating the normal force with respect to separation, the energy of a corrugated sphere and a plate is obtained. Then, differentiating with respect to the phase shift, one finds the lateral Casimir force

$$
F^{l a t}(z, \varphi)=-\frac{4 \pi^{2} R}{\Lambda^{2}} \frac{\partial}{\partial \varphi} \int_{z}^{\infty} d y \int_{0}^{\Lambda} E_{p p}\left(z_{2}-z_{1}\right) d x .
$$

Here all integrals can be calculated explicitly by the use of representation (3) for the separation distance. The result is

$$
F^{l a t}(z, \varphi)=\frac{\pi^{4} R \hbar c}{120 z^{4}} \frac{A_{1} A_{2} \sin \varphi}{\Lambda\left(1-\beta^{2}\right)^{5 / 2}}\left[1+\sum_{n=1}^{4} c_{n, x}\left(\frac{\lambda_{p}}{2 \pi z}\right)^{n}\right]
$$

where $\beta \equiv b(\varphi) / z$ and the coefficients are given by

$$
\begin{aligned}
& c_{1, x}=\frac{4+\beta^{2}}{3\left(1-\beta^{2}\right)} c_{1}, \quad c_{2, x}=\frac{5\left(4+3 \beta^{2}\right)}{12\left(1-\beta^{2}\right)^{2}} c_{2}, \\
& c_{3, x}=\frac{8+12 \beta^{2}+\beta^{4}}{4\left(1-\beta^{2}\right)^{3}} c_{3}, \quad c_{4, x}=\frac{7\left(8+20 \beta^{2}+5 \beta^{4}\right)}{24\left(1-\beta^{2}\right)^{4}} c_{4} .
\end{aligned}
$$

There are also corrections due to the nonzero temperature which can be neglected for the separations reported here [11]. It can be shown that the impact of surface roughness on $F^{\text {lat }}$ is insignificant. 
The experiments are performed using a standard Atomic Force Microscope (AFM) at a pressure below 50 mTorr and at room temperature. A schematic diagram of the experiment is shown in Fig. 1. To implement this experiment a diffraction grating with an uniaxial sinusoidal corrugations of period $\Lambda=1.2 \mu \mathrm{m}$ and an amplitude of $90 \mathrm{~nm}$ was used as the template. In order to obtain perfect orientation and phase between the two corrugated surfaces a special procedure was developed, where the in situ pressure imprinting of the corrugations from the plate onto the gold coated sphere was done. This imprinting procedure required special adaptation of the cantilever. First a polystyrene sphere was attached to the cantilever tip with conductive silver epoxy. Next a $<10 \mu \mathrm{m}$ thick, $100-200 \mu \mathrm{m}$ wide and $0.5 \mathrm{~mm}$ long piece of freshly cleaved mica is attached to the bottom of the sphere with silver epoxy. Then a second polystyrene sphere of $200 \pm 4 \mu \mathrm{m}$ diameter was mounted on the tip of mica with the same silver epoxy. This second sphere is used in the measurement. The cantilever (with mica plate and spheres), corrugated plate and a smooth flat plate (polished sapphire) were then coated with about $400 \mathrm{~nm}$ of gold in a thermal evaporator. A small section of the corrugated plate is also coated with $100 \mathrm{~nm}$ of aluminum (as $A l$ is much harder than gold the imprinting of the corrugations on the sphere is done on this surface). The sphere and the plates are mounted as shown in Fig. 1. As the AFM cantilever is only $320 \mu \mathrm{m}$ long, the addition of the mica plate and the second sphere is necessary to (i) allow access to the central regions of the corrugated plate (ii) reduce the effect of scattered laser light from the edges of the corrugated plate and (iii) increase the sensitivity of the cantilever. Now the sphere is moved over to the region of the corrugated surface coated with $A l$ and the corrugations are imprinted on the gold coating of the surface by pressure using the piezos shown. After this imprinting, the sphere is translated over to the gold coated area of the plate. Extreme care to preserve the parallel orientation of the two corrugations is necessary during this translation, as even a misallignment of $0.1 \mathrm{rad}$ can lead to crossovers of the axis of the two corrugations and destruction of any lateral Casimir force. We used optical beams to monitor the orientation of the cantilever holder with an accuracy of $2 \times 10^{-3}$ radians during the translation process. The amplitude of the corrugation on the plate and the imprinted one on the sphere were measured using the AFM, to be $59 \pm 7 \mathrm{~nm}$ and $8 \pm 1 \mathrm{~nm}$ respectively. This measurement was made after completion of the experiment.

The experimental arrangement in Fig. 1 is so chosen to increase the sensitivity for detection of lateral forces and suppress the effect of the normal Casimir force on the cantilever. Thus a lateral force tangential to the corrugated sphere surface would result in the usual bending of the cantilever in response to the force. Whereas a force acting normal to the sphere and corrugated plate (from the normal Casimir force) would lead to the torsional deflection (rotation) of the cantilever. The torsional spring constant of this cantilever $k_{t o r}$ is much greater than the bending spring constant $k_{\text {ben }}$, making it much more sensitive to detecting the lateral Casimir force, while simultaneously suppressing the effect of the normal Casimir force. The calibration of the cantilever ( $k_{t o r}$ and $\left.k_{b e n}\right)$ and the measurement of the residual potentials between the sphere and plate is done by electrostatic means in a manner similar to that in Refs. [7] 10]. Here the sphere is kept grounded and various voltages are applied to the plates. The normal electrostatic force between the corrugated sphere and plate is given by:

$$
F^{e l}(z, \varphi)=-\pi R \varepsilon_{0} \frac{\left(V_{1}-V_{0}\right)^{2}}{z} \frac{1}{\sqrt{1-\beta^{2}}},
$$

where as before $z$ is the total distance between the zero corrugation levels on the corrugated surfaces. $V_{1}$ are the voltages applied on the corrugated plate and $V_{0}$ is the residual potential on the grounded sphere. If $V_{1}$ is applied to the corrugated plate, the electrostatic force on the sphere leads to the torsional rotation of the cantilever. From the electrostatic force at different $V_{1}$ we measure the torsional spring constant $k_{t o r}=0.138 \pm 0.005 \mathrm{~N} / \mathrm{m}$ and $V_{0}=-0.135 \mathrm{~V}$. Similarly if the sphere is brought close to the bottom smooth plate and voltages $V_{1}$ are applied to the bottom plate (here in Eq. (7) $A_{1}=A_{2}=\beta=0$ due to the smooth surfaces), the electrostatic force leads to the normal bending of the cantilever. From the force at different voltages $V_{1}$ we again measure the normal spring constant $k_{\text {ben }}=0.0052 \pm 0.0001 \mathrm{~N} / \mathrm{m}$. Note that $k_{t o r} \gg k_{\text {ben }}$ is required for isolation and detection of the lateral Casimir force. The piezo extension with applied voltage was calibrated by optical interferometry [31].

Next the lateral Casimir force is measured. The residual potential difference between the corrugated sphere and plate is compensated by application of voltage $V_{0}$ to the corrugated plate. The corrugated plate is mounted on two piezos that allow independent movement of the plate in the vertical and the horizontal directions with help of a $x$-piezo and $z$-piezo respectively. Movement in the $x$ direction with the $x$-piezo is necessary to achieve lateral phase shift $\varphi$ between the corrugations on the sphere and the plate. Independent movement in the $z$ direction is necessary for control of the surface separation between the corrugated sphere and plate. Also, the corrugated plate could be mounted with a small but non-zero tilt away from the vertical. This would lead to changes in surface separation during lateral translation of the plate in the $x$ direction. To prevent this a small correction voltage is applied to the $z$-piezo synchronous with the lateral translation in $x$ direction, to keep the surface separation distance constant. 
At the start of lateral force measurement, the sphere is brought close to the corrugated plate and the separation distance is kept fixed. To measure the lateral force $F^{\text {lat }}$ as a function of the phase $\varphi$, the corrugated plate is moved in the $x$-direction in $0.46 \mathrm{~nm}$ steps using the $x$-piezo and the lateral force is measured at each step. This is repeated 60 times and the average lateral force at each step is recorded. The average lateral force measured is shown as the solid squares in Fig. 2. The sinusoidal oscillations expected from Eq. (6) as a function of the phase difference between the two corrugations are clearly observed. Also the periodicity of the lateral force oscillation is in agreement with corrugation period of the plate. A sine curve fit to the observed data is shown as the solid line and corresponds to an amplitude of $3.2 \times 10^{-13} \mathrm{~N}$. From Eq. (6) this corresponds to a separation distance of $z=221 \pm 2 \mathrm{~nm}$ between the two corrugated surfaces. The mean quadratic error of the average lateral force amplitude is $\sigma_{\bar{A}}=0.15 \times 10^{-13} \mathrm{~N}$. Systematic error is around $5 \%$ leading to the absolute error $\Delta_{A}^{(s)}=0.16 \times 10^{-13} \mathrm{~N}$. Using the value of Student coefficient $t_{0.95,60}=2$ one obtains for the confidence interval $\Delta_{A}=0.46 \times 10^{-13} \mathrm{~N}$ with a $95 \%$ confidence probability. The resulting precision of the amplitude measurement is around $15 \%$.

Next the separation between the sphere and corrugated plate is increased by $12 \mathrm{~nm}$ with the $z$-piezo and the measurement is repeated. The average measured amplitude of lateral force of $2.6 \times 10^{-13} \mathrm{~N}$ corresponds from Eq. (6) to $z=233 \pm 2 \mathrm{~nm}$ consistent with the $12 \mathrm{~nm}$ increase in the separation distance. Thus the measured lateral force is in agreement with the complete theory taking into account the conductivity corrections. The separation distance is increased in $12 \mathrm{~nm}$ steps and the lateral force is measured two more times. The amplitude of the measured forces was found to be consistent with the corresponding separation distances. In Fig. 3 a log-log plot of the amplitude of measured lateral force as a function of the separation distance is shown as solid squares. Here the separation distance of $221 \mathrm{~nm}$ determined above is used for the closest point. The remainder of the points are fixed by the $12 \mathrm{~nm}$ step increase in the separation distance. A linear fit to the data yields a slope of $4.1 \pm 0.2$ consistent with the inverse fourth power $z$ dependence of the lateral force expected from Eq. (6) (corrections to this dependence are rather small as the maximal value of $\beta=0.3$ ). Note that a lateral force resulting from electrostatic effects would lead to an inverse second power $z$ dependence.

In conclusion, we have made the first measurement of the lateral Casimir force. It was measured between a corrugated plate and corrugated sphere using an AFM. Exact allignment of the corrugations on the sphere and plate was achieved by a special imprinting technique. The measured lateral Casimir force is sinusoidal with a periodicity corresponding to the corrugation period. It was shown to have an inverse fourth power distance dependence which is in agreement with the theory taking into account the conductivity corrections. This demonstration of the lateral Casimir force should open new opportunities for the application of the Casimir effect in microelectromechanical systems.

This work is supported by a National Science Foundation Nanoscale Exploratory Research grant and the National Institute for Standards and Technology through a Precision Measurement Grant. G.L.K. and V.M.M. were also supported by CNPq.

[1] H. B. G. Casimir, Proc. K. Ned. Akad. Wet. 51, 793 (1948).

[2] P. W. Milonni, The Quantum Vacuum (Academic Press, San Diego, 1994).

[3] V. M. Mostepanenko and N. N. Trunov, The Casimir Effect and its Applications (Clarendon Press, Oxford, 1997).

[4] M. J. Sparnaay, Physica 24, 751 (1958).

[5] P. H. G. M. van Blockland and J. T. G. Overbeek, J. Chem. Soc. Faraday Trans. 74, 2637 (1978).

[6] S. K. Lamoreaux, Phys. Rev. Lett. 78, 5 (1997).

[7] U. Mohideen and A. Roy, Phys. Rev. Lett. 81, 4549 (1998).

[8] A. Roy, C.-Y. Lin, and U. Mohideen, Phys. Rev. D 60, 111101(R) (1999).

[9] B. W. Harris, F. Chen, and U. Mohideen, Phys. Rev. A 62, 052109 (2000).

[10] A. Roy and U. Mohideen, Phys. Rev. Lett. 82, 4380 (1999).

[11] M. Bordag, U. Mohideen, and V. M. Mostepanenko, Phys. Rep. 353, 1 (2001).

[12] M. Bordag, B. Geyer, G. L. Klimchitskaya, and V. M. Mostepanenko, Phys. Rev. D 58, 075003 (1998); 60, 055004 (1999); 62, 011701(R) (2000).

[13] V. M. Mostepanenko and M. Novello, Phys. Rev. D 63, 115003 (2001).

[14] E. Fischbach, D. E. Krause, V. M. Mostepanenko, and M. Novello, Phys. Rev. D 64, 075010 (2001).

[15] H. B. Chan, V. A. Aksyuk, R. N. Kleiman, D. J. Bishop, and F. Capasso, Science, 291, 1941 (2001).

[16] H. B. Chan, V. A. Aksyuk, R. N. Kleiman, D. J. Bishop, and F. Capasso, Phys. Rev. Lett, 87, 211801 (2001).

[17] E. Buks and M. L. Roukes, Phys. Rev. B 63, 033402 (2001). 
[18] Yu. S. Barash, Sov. Radiophys. 16, 945 (1975).

[19] S. J. van Enk, Phys. Rev. A 52, 2569 (1995).

[20] R. Golestanian and M. Kardar, Phys. Rev. Lett. 78, 3421 (1997).

[21] R. Golestanian and M. Kardar, Phys. Rev. A 58, 1713 (1998).

[22] T. Emig, A. Hanke, and M. Kardar, cond-mat/0106028.

[23] L. S. Levitov, Europhys. Lett. 8, 499 (1989).

[24] J. S. Hoye and I. Brevik, Physica A 181, 413 (1992).

[25] I. E. Dzyaloshinskii, E. M. Lifshitz, and L. P. Pitaevskii, Sov. Phys. - Usp. (USA) 4, 153 (1961).

[26] J. Schwinger, L. L. DeRaad, and K. A. Milton, Ann. Phys. (N.Y.) 115, 1 (1978).

[27] V. B. Bezerra, G. L. Klimchitskaya, and V. M. Mostepanenko, Phys. Rev. A 62, 014102 (2000).

[28] G. L. Klimchitskaya, A. Roy, U. Mohideen, and V. M. Mostepanenko, Phys. Rev. A 60, 3487 (1999).

[29] J. Blocki, J. Randrup, W. J. Swiatecki, and C. F. Tsang, Ann. Phys. 105, 427 (1977).

[30] G. Barton, private communication.

[31] F. Chen and U. Mohideen, Rev. Sci. Instr. 72, 3100 (2001). 
Figure 1: Schematic of experimental setup. For clarity, the size of the corrugations have been exaggerated and the additional mica plate and the first sphere attached to the cantilever are not shown. The $x$-piezo and $z$-piezo are independent.

Figure 2: The average measured lateral Casimir force as a function of the lateral displacement of the corrugated plate is shown as solid squares. The solid line is the best fit sine curve to the data leading to a lateral force amplitude of $3.2 \times 10^{-13} \mathrm{~N}$.

Figure 3: log-log plot of the measured lateral Casimir force amplitude as a function of the surface separation distance is shown as solid squares. The slope of the straight line fit is $4.1 \pm 2$. 
Fig. 1

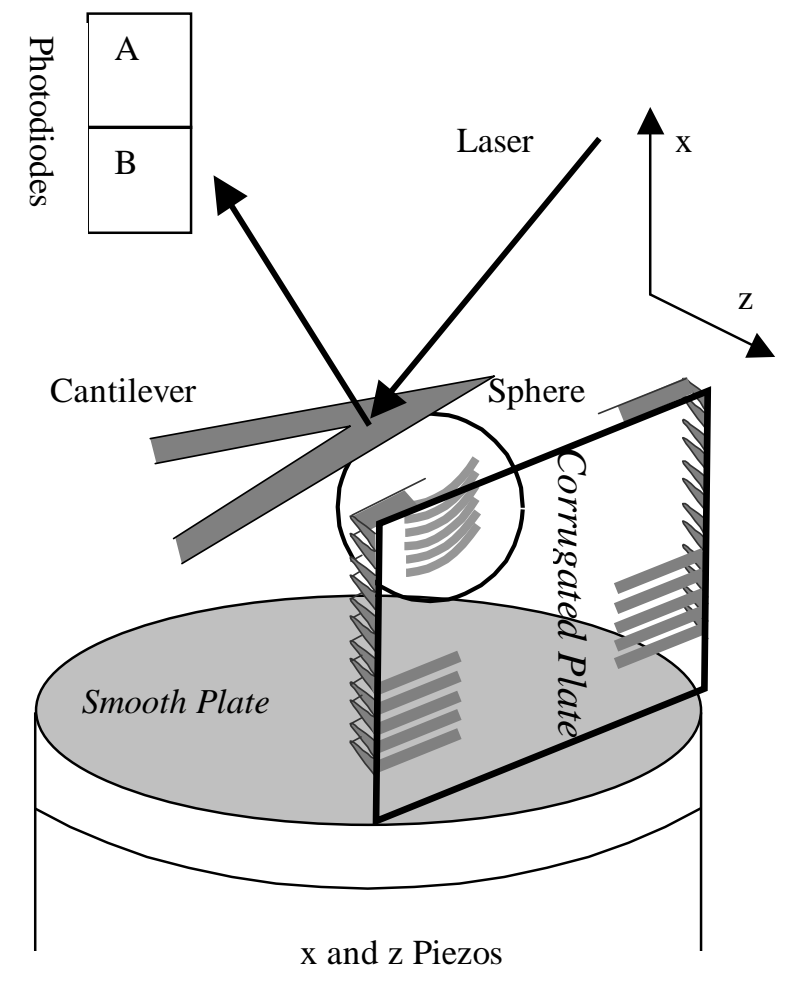


Fig 2

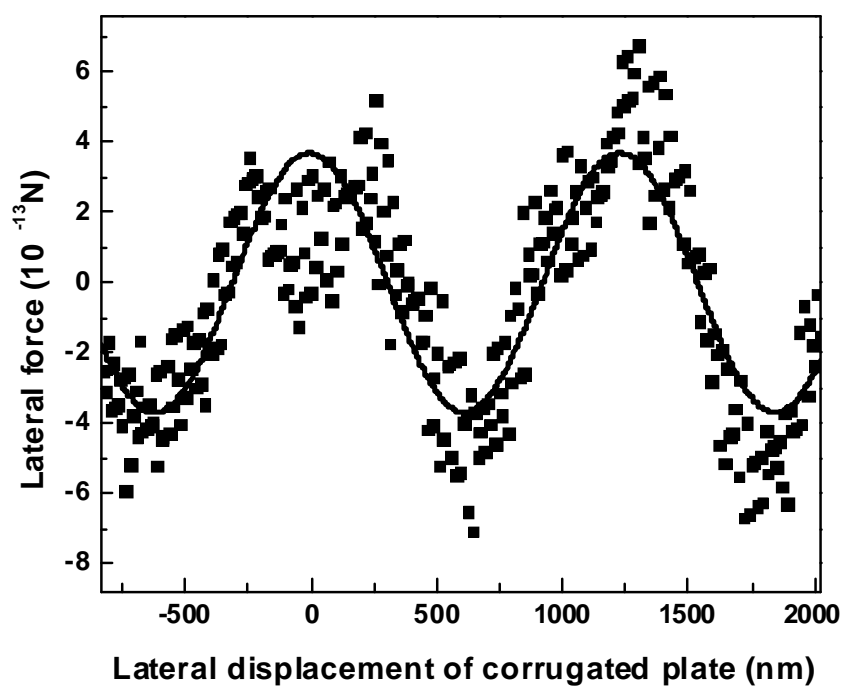


Fig. 3

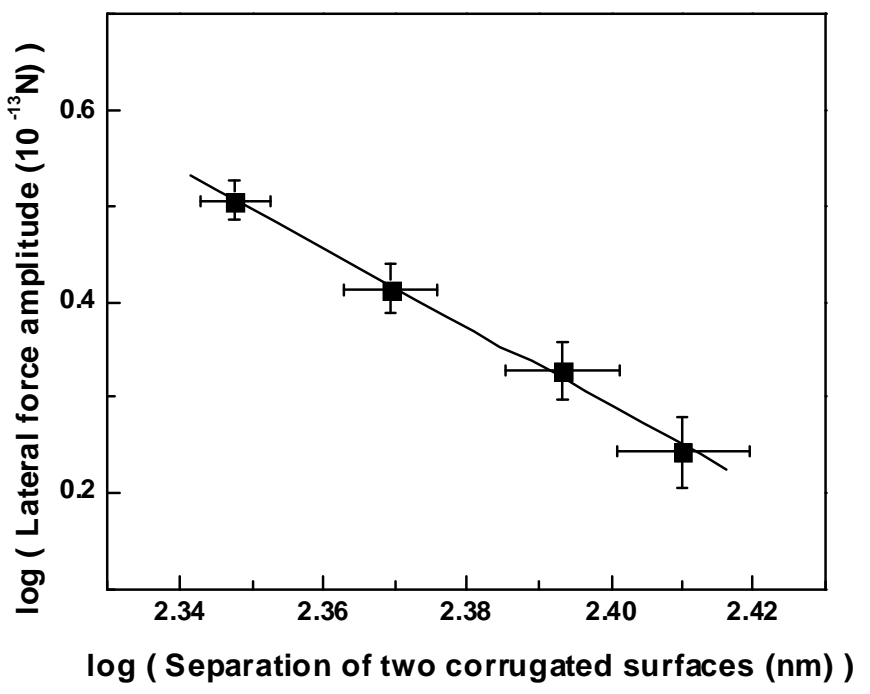

